\title{
Association of Vistech contrast sensitivity and visual field findings in glaucoma
}

\author{
William E Sponsel, Kathleen L DePaul, James F Martone, M Bruce Shields, Anita R Ollie, \\ William C Stewart
}

University of WisconsinMadison Department of Ophthalmology, USA

W E Sponsel Institute, Los Angeles, California

J F Martone

Duke University Eye Center, Durham, North Carolina M B Shields

A R Ollie

Storm Eye Institute, Charleston, South Carolina

W C Stewart

Correspondence to: William E Sponsel, MD, Department of Ophthalmology, F4/3 CSC 600 Highland Avenue, Madison, WI 53792, USA

Accepted for publication Madison, Wisconsin,

K L DePaul

Estelle Doheny Eye 30 May 1991

\section{Abstract}

Single eye visual fields and contrast sensitivity were assessed in 60 subjects, who were being followed up in a glaucoma clinic for manifest glaucoma or a suspicion of glaucoma because of raised intraocular pressure. The Fieldmaster 5000 (static/kinetic perimeter) was used for the visual fields, and a Vistech wall chart sine wave grating test was used for contrast sensitivity measurements. The subjects were divided into three groups - defect (D), suspect (S) and normal $(\mathbf{N})$ - on the basis of their perimetric findings by subjective grading of 16 perimetric scoring categories for each visual field. The mean Vistech sensitivity levels were not found to be significantly different between the $D, S$, and $\mathbf{N}$ field subgroups at any of the five spatial frequencies provided on the test charts $(1 \cdot 5,3$, 6,12 , and 18 cycles per degree). Complex algorithms combining results from two or more spatial frequencies also failed to yield any significant differences between the groups. Diagnostic sensitivity and specificities relating Vistech contrast sensitivity findings to groups $N$ and $D$ never concomitantly exceeded $60 \%$.

Measurement of the visual fields is the principal method for confirming the presence and progression of glaucoma. Despite recent advances in computerised visual field analysis, perimetric studies remain time consuming and demand complex subjective interpretation. Psychophysical research has revealed abnormalities in contrast sensitivity in association with glaucoma and ocular hypertension..$^{1-6}$ Recently

Table 1 Criteria used for interpretation of visual fields

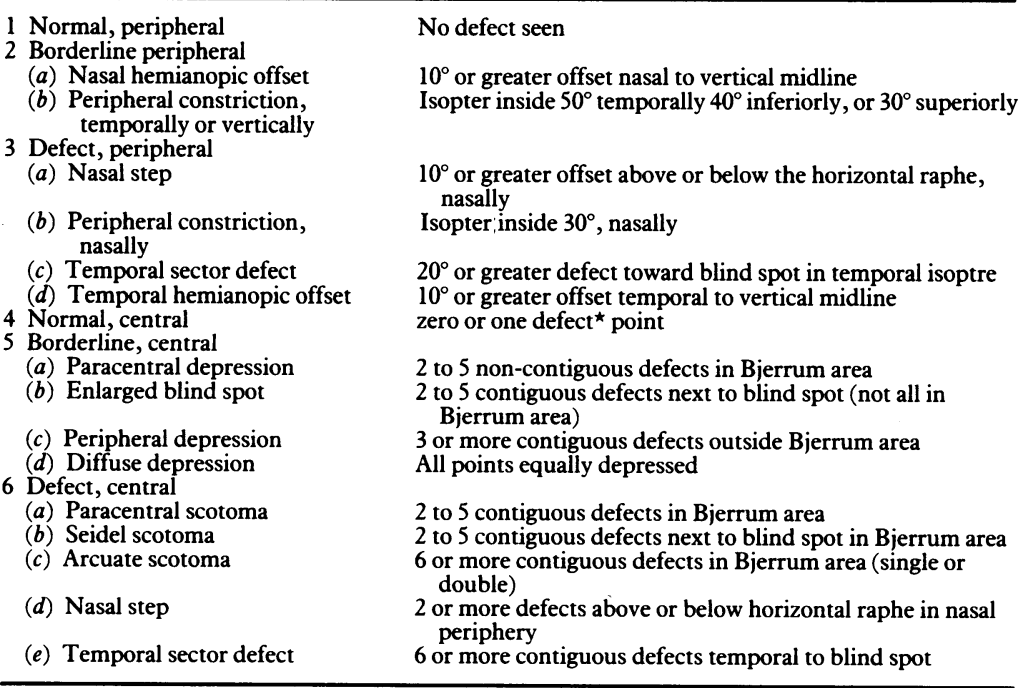

^Defect refers to depression of $6 \mathrm{~dB}$ or more below age-related retinal threshold for point being tested.
Table 2 Comparison of Vistech line scores A through $E^{\star}$ $(1 \cdot 5,3,6,12$, and 18 cycles per degree) for normal, suspect and defect groups

\begin{tabular}{llcr}
\hline & Normal & Suspect & Defect \\
\hline Number & 16 & 13 & 31 \\
Line score A & $5 \cdot 1(0 \cdot 2)$ & $5 \cdot 0(0 \cdot 2)$ & $5 \cdot 0(1 \cdot 0)$ \\
Line score B & $5 \cdot 5(0 \cdot 3)$ & $5 \cdot 7(0 \cdot 2)$ & $5 \cdot 4(0 \cdot 1)$ \\
Line score C & $4 \cdot 5(0 \cdot 4)$ & $4 \cdot 8(0 \cdot 3)$ & $4 \cdot 3(0 \cdot 3)$ \\
Line score D & $3 \cdot 3(0 \cdot 6)$ & $3 \cdot 8(0 \cdot 5)$ & $3 \cdot 5(0 \cdot 3)$ \\
Line score E & $2 \cdot 8(0 \cdot 5)$ & $2 \cdot 8(0 \cdot 5)$ & $2 \cdot 4(0 \cdot 3)$ \\
\hline
\end{tabular}

^Standard errors are in parentheses. No significant differences between the three groups were found.

clinical methods for presenting static contrast gratings on wall charts have been introduced. ${ }^{78}$ One of these is the Vistech sine wave grating method. Bron has reported that oscilloscopic and Vistech methods discriminate glaucomatous from ocular hypertensive eyes equally well. ${ }^{9}$ The purpose of the present study was to assess the association between visual fields, categorised by traditional criteria, and concomitant Vistech contrast sensitivity measurements.

\section{Materials and methods}

The study population consisted of subjects who were being followed up in the Glaucoma Clinic of Duke University Eye Center for manifest glaucoma or a suspicion of glaucoma on the basis of intraocular pressure elevation. Visual fields were assessed with the Fieldmaster 5000 (static/ kinetic perimeter; Bausch and Lomb, Rochester, NY), and contrast sensitivity was measured under conditions of constant illumination with Vistech sine wave gratings (Vistech Consultants, Dayton, Ohio). The Fieldmaster 5000, the details of which have been previously described, ${ }^{10}$ is a fully automated perimeter which measures the peripheral field with a kinetic target and the central $30^{\circ}$ with static targets. In the present

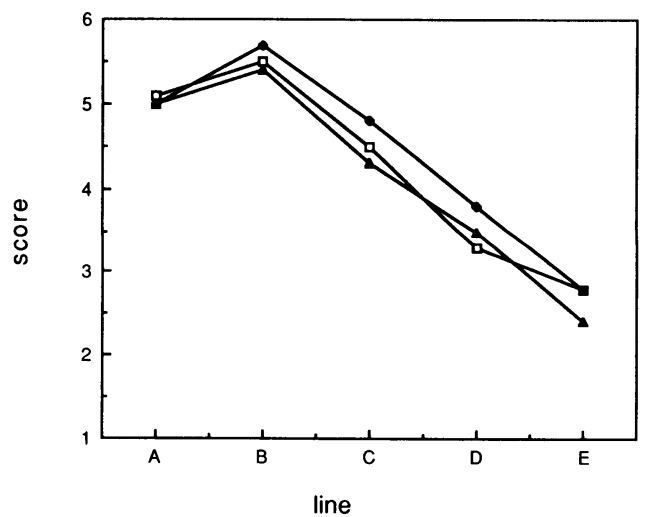

Figure 1 Average Vistech contrast sensitivity line scores for normal, suspect, and defect groups. Lines $A-E$ correspond to five static spacial frequencies: $1 \cdot 5,3,6,12$, and 18 cycles per degree. Normal $\square$. Suspect 
Figure 2 Receiver operator characteristic $(R O C)$ curve showing Vistech line B score ( 3 cycles per degree) as a detector of glaucomatous field loss. Criteria for abnormal classification are indicated along the curve.

Figure 3 ROC curve showing Vistech line $C$ score (6 cycles per degree) as a detector of glaucomatous field loss. Criteria for abnormal classification are indicated along the curve.
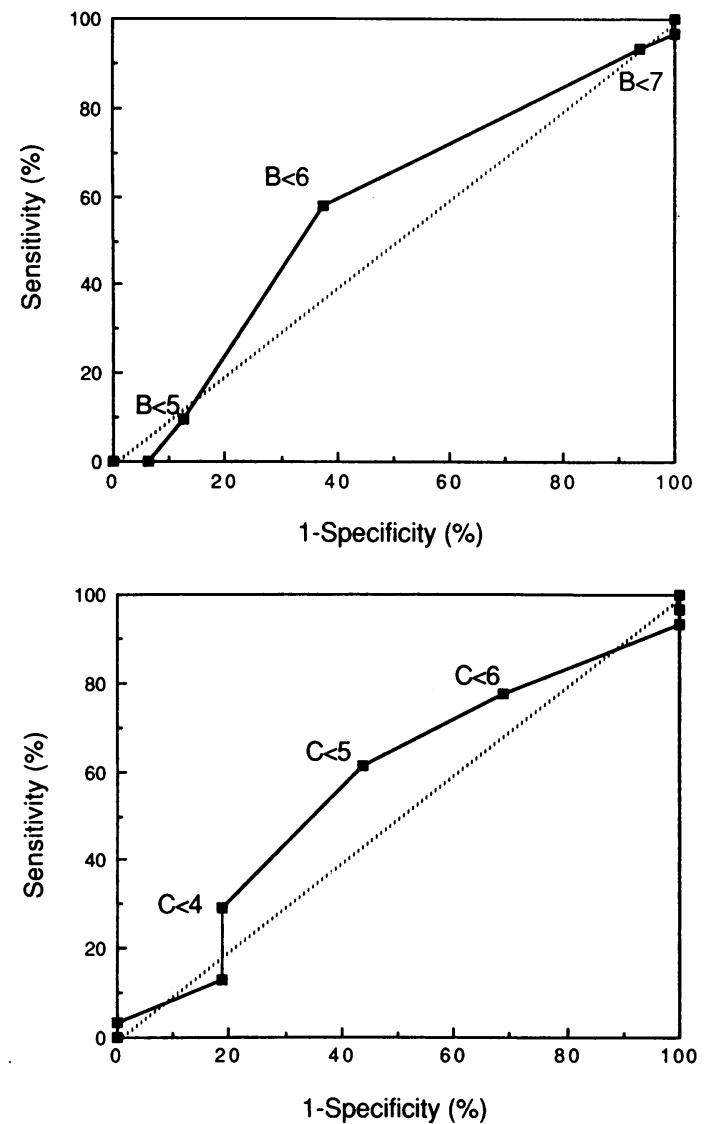

study the central, static points were first tested with a supratheshold stimulus, followed by full thresholding of all points missed on the initial presentation, and the peripheral field was measured with a single isopter. The Vistech wall chart system provides a rapid score on an integral scale from 0 to 8 , corresponding to the number of patterns correctly identified, for each of five static spatial frequencies. These frequencies, $1 \cdot 5,3,6,12$, and 18 cycles per degree, are defined as lines $\mathrm{A}$ through $\mathrm{E}$ respectively.

Visual fields were evaluated in masked fashion by glaucoma clinicians (MBS and WCS) into $\operatorname{defect}(\mathrm{D})$, suspect $(\mathrm{S})$, and normal $(\mathrm{N})$ categories on the basis of detailed subjective criteria which involved pass/fail grading into a total of 16 separate subcategories (Table 1). Patients assigned to group D included those with definitive glaucomatous changes or $>2$ borderline abnormalities. Group $\mathrm{N}$ included those subjects with no abnormal findings, and group $S$ included the remainder. When both fields from a single subject fell into the same rating group, one was randomly selected for analysis. Otherwise the eye with the greater defect was included, so that only one eye from each subject was considered. All eyes under study had visual acuities of $20 / 40$ or better. Differences between the two groups were analysed by the unpaired $t$ test. Sensitivites and specificities were determined for a variety of criteria, with Vistech scores being used as detectors of glaucomatous field loss.

Results

Sixty patients were included in the study, 31 in group D, 13 in group S, and 16 in group N. The mean ages for the $D, S$, and $N$ subgroups were:
Table 3 Sensitivity and specificity values using Vistech line scores $B$ ( 3 cycles per degree) and $C$ ( 6 cycles per degree) as detectors of glaucomatous field loss

\begin{tabular}{lll}
\hline Abnormal classification & Sensitivity & Specificity \\
\hline B $<6$ & $58 \cdot 1 \%$ & $62 \cdot 5 \%$ \\
C $<5$ & $61 \cdot 3 \%$ & $56 \cdot 3 \%$ \\
C $<5$ and B $<6$ & $48 \cdot 4 \%$ & $68 \cdot 8 \%$ \\
C $<5$ or B $<6$ & $71 \cdot 0 \%$ & $50 \cdot 0 \%$ \\
average $(B$ and C) $<5$ & $68 \cdot 8 \%$ & $51 \cdot 6 \%$ \\
\hline
\end{tabular}

54 (range 25-76), 55 (range 29-78), and 53 (range 25-72) respectively. Table 2 and Fig 1 show the average contrast sensitivity scores for the three groups. Mean scores of the D, S, and N field groups did not differ significantly at any of the five spatial frequencies provided on the test charts. Complex algorithms looking for midrange deficit - that is, average $(A, B)-C ; A$-average $(\mathrm{B}, \mathrm{C}) ; \mathrm{A}+\mathrm{D}-\mathrm{B} ; \mathrm{A}+\mathrm{D}-\mathrm{C} ; \mathrm{A}+\mathrm{B}-(\mathrm{C}+\mathrm{D}) ; \mathrm{A}+$ $\mathrm{D}-(\mathrm{B}+\mathrm{C}) ; \mathrm{B}-\mathrm{C} ; \mathrm{A}-\mathrm{C} ; \mathrm{A}-\mathrm{B})-$ also failed to yield any significant mean differences between the groups. Diagnostic sensitivity/specificity (receiver operator characteristic; ROC) curves relating Vistech contrast sensitivity findings to perimetry groups $\mathrm{N}$ and $\mathrm{D}$ are shown in Figs 2 and 3 for lines B and C ( 3 and 6 cycles per degree). These data suggest that $V$ istech contrast sensitivity scores of $B<6$ or $C<5$ offer the greatest combination of diagnostic sensitivity and specificity. A looser cutoff point $(B<7$ or $\mathrm{C}<6$ ) would include many normal subjects, while a more rigid cutoff limit $(B<5$ or $C<4)$ would miss many glaucomas. Vistech results at or below the latter levels, however, would provide a reasonable indication of pathology, with false positive rates of $<20 \%$. Table 3 includes sensitivity/specificity values for classification criteria involving lines B and C. Three criteria produce sensitivity and specificity values concomitantly greater than $50 \%: B<6, C<5$, and average $(B, C)<5$. None produced sensitivity and specificity values concomitantly exceeding $60 \%$.

\section{Discussion}

The sensitivity/specificity values obtained in the present study for contrast sensitivity scores are low, with the best association with subjectively classified visual fields seen at 3 and 6 cycles per

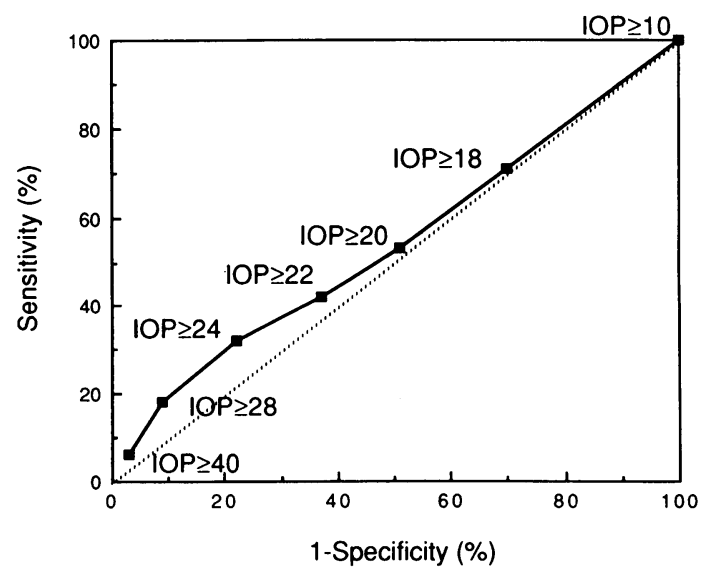

Figure 4 ROC curve showing intraocular pressure (IOP in $\mathrm{mmHg}$ ) as a detector of glaucomatous field loss. Criteria for abnormal classification are indicated along the curve. Adapted from Hill ${ }^{\prime \prime}$ based on data from Daubs and Crick. ${ }^{\prime 2}$ 
degree. These sensitivity/specificity curves are nevertheless more definitive than published associations between intraocular pressure and visual fields (Figure 4). ${ }^{11}$ The findings presented here are consistent with those of Adams et $a l^{13}$ who found that mean contrast sensitivities of glaucomatous subjects were significantly lower $(p<0.02)$ at all frequencies but were within one standard deviation of those of the normal controls.

While measurements of contrast sensitivity involve the perimacular region of the retina, classic glaucomatous field loss characteristically occurs more peripherally. Recent work in this laboratory (UW-Madison) reveals a significant association $(p<0.05)$ between the bilateral asymmetry of Vistech contrast sensitivity scores (at 3 and 6 cycles per degree) and the asymmetry of visual field indices obtained by Humphrey automated perimetry in glaucomatous patients. ${ }^{14}$ In the present study, however, asymmetry of Vistech scores between the two eyes did not correlate significantly with the presence of a visual field defect in either eye. Together these studies demonstrate the importance of distinguishing between correlation of contrast sensitivity with glaucomatous change and its actual diagnostic utility.

The use of subjective visual field assessments as the standard for comparison in this study does not preclude the possibility that the Vistech system may elicit some early glaucomatous changes to which perimetry remains insensitive. If so, however, such static contrast changes must occur frequently among normal individuals as well. Thresholding only defective areas in the present study may have slightly reduced the sensitivity of the visual field testing, though a study using the Humphrey Analyzer has revealed differences in subjective interpretation between paired full thresholding and quantification of defects in only 18 of 104 fields. ${ }^{\text {is }}$ It is possible that modifications of existing contrast sensitivity testing methods may increase their diagnostic utility for glaucoma detection. Such adaptations might include the use of temporal modulation and/or opponent colour contrast paradigms within a rapid and clinically amenable testing format. ${ }^{13}$ 16-27

1 Arden GB, Jacobson J. A simple grating test for contras sensitivity - glaucoma screening. Invest Ophthalmol Vis Sci 1978; 17: 23-32.
2 Stamper RL, Hsu-Winges C, Sopher M. Arden contrast sensitivity testing in glaucoma. Arch Ophthalmol 1982;100: 947-50.

3 Stamper RL. The effect of glaucoma on central visual function. Trans Am Ophthalmol Soc 1984; 82: 792-826.

4 Moskowiz A, Sokol S, Bardenstein D, et al. Performance of ocular hypertension and glaucoma patients on high and low contrast charts (abstract). Invest Ophthalmol Vis Sci 1985; 26 contrast char

5 Ross JE, Bron AJ, Clarke DD. Contrast sensitivity and visual disability in chronic simple glaucoma. $\mathrm{Br} \mathcal{F}$ Ophthalmo 1984; 68: 821-7.

6 Ross JE, Bron AJ, Reeves BC and Emmerson PG. Detection of optic nerve damage in ocular hypertensives. $\mathrm{Br} F$ Ophthalmo 1985; 69: 897-903.

7 Ginsberg AP. A new contrast sensitivity vision test chart. Am $\mathcal{F}$ Optom Physiol Opt 1984; 61: 403-7.

8 Regan D, Neima D. Low-contrast letter charts in early diabetic retinopathy, ocular hypertension, glaucoma, and diabetic retinopathy, ocular hypertension, glaucoma,

9 Bron AJ. Contrast sensitivity changes in ocular hypertension and early glaucoma (summary). Surv Ophthalmol 1989; 33 (suppl): 405-6.

10 Stewart WC, Shields MB, Ollie AR. Peripheral visual field testing by automated kinetic perimetry in glaucoma. Arch Ophthalmol 1988; 106; 202-6.

11 Hill AR. Making decisions in ophthalmology. In: Osborne N Chader G, eds. Progress in retinal research. New York: Pergamon, 1987; 6: 227

12 Daubs J, Crick RP. Epidemiological analysis of King's College Hospital glaucoma data. Res Clin Forums 1980; 2: 41-59.

13 Adams AJ, Heron F, Husted R. Clinical measures of central visual function in glaucoma and ocular hypertension. Arch Ophthalmol 1987; 105; 782-7.

14 Sponsel WE, DePaul KL, Kaufman PL. Correlation of visual function and retinal leucocyte velocity in glaucoma. $A m \mathcal{F}$ Ophthalmol 1990; 109: 49-54

15 Stewart WC, Shields MB, Ollie AR. Full threshold versus quantification of defects for visual field testing in glaucoma. Graefes Arch Clin Exp Ophthalmol 1989; 227; 51-4.

16 Stamper RL. Psychophysical testing in glaucoma. Surv Ophthalmol 1989; 33 (suppl): 309-18.

17 Atkin A, Bodis-Wollner I, Podos SM, Wolkstein M, Mylin L, Nitzberg S. Flicker threshold and pattern VEP latency in ocular hypertension and glaucoma. Invest Ophthalmol Vis Sci 1983; 24: 1524-7.

18 Atkin A, Wolkstein M, Bodis-Wollner I, Anders M, Kels B, Podos SM. Interocular comparison of contrast sensitivities in glaucoma patients and suspects. Br $\mathcal{F}$ Ophthalmol 1980; 64: 858-62.

19 Atkin A, Bodis-Wollner I, Wolkstein M, Moss A, Podos SM. Abnormalities of central contrast sensitivity in glaucoma. Am F Ophthalmol 1979; 88: 205-11.

20 Gündüz K, Arden GB, Perry S, Weinstein GW, Hitchings RA. Color vision defects in ocular hypertension and glaucoma: quantification with a computer-driven color television system. Arch Ophthalmol 1988;106: 929-35.

21 Motolko MA, Phelps CD. Contrast sensitivity in asymmetric glaucoma. Int Ophthalmol 1984; 7: 45-50.

22 Regan D, Neima D. Balance between pattern and flicker sensitivities in the visual fields of ophthalmological patients. Br F Ophthalmol 1984; 68: 310-5.

23 Sample PA, Boynton RM, Weinreb RN. Isolating the color vision loss in primary open-angle glaucoma. Am $\mathcal{f} O$ phthalmol 1988; 106: 686-91.

24 Stelmach LB, Drance SM, Di Lollo V. Two-pulse temporal resolution in patients with glaucoma, suspected glaucoma and in normal observers. Am $\mathcal{F}$ Ophthalmol 1986; 102: 61720.

25 Tyler CW. Specific defects of flicker sensitivity in glaucoma and ocular hypertension. Invest Ophthalmol Vis Sci 1981; 20: 204-12.

26 Tytla ME, Trope GE, Buncic JR. Flicker sensitivity in treated ocular hypertension Ophthalmology 1990; 97: 36-43.

27 Marx MS, Bodis-Wollner I, Lustgarten JS, Podos SM. Electrophysiological evidence that early glaucoma affects foveal vision. Doc Ophthalmol 1987; 67: 281-301. 